\title{
MANEJO DE LAS COMPETENCIAS EMOCIONALES FRENTE A LA TRANSFORMACIÓN CURRICULAR
}

\author{
Norelit Gutiérrez \\ noregutierrezt@hotmail.com \\ Jairo Villasmil \\ jaravife@hotmail.com \\ Nicolás Rodríguez \\ nicolas.rodriguez702@gmail.com \\ Universidad Nacional Experimental Francisco de Miranda \\ Recibido: 04 de mayo de 2016 \\ Aprobado: 31 de mayo de 2016
}

\begin{abstract}
RESUMEN
Este artículo tiene como propósito, conocer cómo manejar las emociones frente a los cambios curriculares en instituciones universitarias. En una formación universitaria, la inteligencia emocional y el manejo de la misma es indispensable, por lo tanto, es importante que el que dirige, oriente y fortalece las relaciones de los actores educativos, tanto en lo personal como en lo social, debe ser capaz de manejar los esfuerzos colectivos hacia una continua transformación y adaptación a las exigencias sociales. En las personas racionales, las emociones no solo tiene un papel más determinante en la toma de decisiones, sino que además el individuo puede tener el control de la impulsividad, persistencia, motivación, empatía y destrezas sociales. Cuando un individuo conoce sus propias emociones, quiere decir que tiene la capacidad de conocerse a sí mismo. Goleman (1995) defiende el planteamiento en el que se afirma que existen dos mentes, una racional y una emocional, y que las formas de conocimientos son distintas para cada caso y cuando trabajan de forma concatenada, interconectando los diferentes conocimientos, la inteligencia emocional crece al igual que la capacidad intelectual.
\end{abstract}

Descriptores: Transformación Curricular, Inteligencia Emocional, Competencias Emocionales, Emocionalidad, Capacidad Intelectual 


\title{
MANAGEMENT OF EMOTIONAL COMPETENCES IN FRONT OF CURRICULAR TRANSFORMATION
}

\begin{abstract}
SUMMARY
This article aims to learn how to handle emotions in the face of curricular changes in university institutions. In a university formation, the emotional intelligence and the management of the same is indispensable, therefore, it is important that the one that directs, orientates and strengthens the relations of the educational actors, in the personal as in the social, must be able to manage the collective efforts towards a continuous transformation and adaptation to the social demands. In rational people, emotions not only have a more decisive role in decision making, but also the individual can have control of impulsiveness, persistence, motivation, empathy and social skills. When an individual knows their own emotions, it means that they have the ability to know themselves. Goleman (1995) defends the approach that states that there are two minds, one rational and one emotional, and that the forms of knowledge are different for each case and when they work in a concatenated way, interconnecting different knowledge, emotional intelligence grows as well as intellectual capacity.
\end{abstract}

Keywords: Curricular Transformation, Emotional Intelligence, Emotional Competences, Emotionality, Intellectual Capacity

\section{INTRODUCCIÓN}

Para llevar a cabo una transformación curricular, se debe tomar en cuenta todos los aspectos que afecta de una $u$ otra forma el cambio curricular. La transformación no sólo debe ser en papel, en la infraestructura, en dotaciones o adaptaciones a las nuevas teorías de aprendizaje o a las exigencias y demanda laborales, es también un cambio en la actitud y concepción de cada miembro o actor educativo con respecto al cambio, donde cada uno tiene una función, un rol, un deber que cumplir dentro de la institución educativa, por ende cada miembro es importante y debe ser tomado en cuenta para así lograr con éxito una formación basada en desarrollar, capacidades, destrezas, habilidades, virtudes, ética y sentido crítico en los futuros egresados; para que puedan llegar a ser los 
profesionales competitivos que cumplan con las exigencias y demandas empresariales y sociales.

Así mismo, en el tema de la emocionalidad, es importante destacar la inteligencia emocional, propuesto por Goleman (1995), en el que señala que "es la capacidad que un individuo posee para conocer e interpretar las emociones de las diferentes personas, de manera externas e internamente" (p.97). Por lo tanto, una persona con "inteligencia emocional" tiene la capacidad de conocer y controlar no sólo sus propias emociones, sino además las de otras personas y hasta las de un grupo de personas.

En el estudio de la inteligencia emocional, se encuentran cinco "competencias" consideradas básicas que se pueden aprender, y de ser así, éstas habilitarían y darían capacidades a las personas, para ser mejores consigo mismas y con los demás.

Tomando en cuenta el modelo de Daniel Goleman, de las competencias de la inteligencia emocional se derivan las "intrapersonales" e "interpersonales". La primera categoría trata de las emociones propias de cada individuo, la segunda se refiere a las interacciones emocionales entre dos o más personas.

Las competencias de la inteligencia emocional, señaladas por Goleman (1995) son:

1. Conocer las propias emociones: El principio de Sócrates "conócete a ti mismo" se refiere a esta pieza clave de la inteligencia emocional: tener conciencia de las propias emociones; reconocer un sentimiento en el momento en que ocurre. Reconocernos incapaz en este sentido nos dejaría en manos de las emociones incontroladas.

2. Manejar las emociones: La habilidad para controlar los propios sentimientos con la finalidad de que éstos se expresen de manera apropiada, se basa en la toma de conciencia de las propias emociones. Es la habilidad para suavizar los sentimientos de ira, furia o irritabilidad, ésta es esencial en las relaciones interpersonales. 


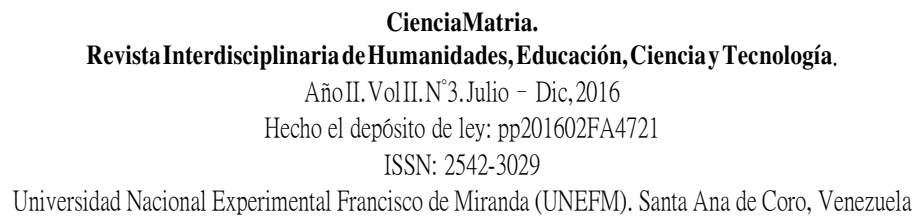

3. Motivarse a sí mismo: Una emoción tiende a estimular hacia una acción. Por lo que, la emoción y motivación están estrechamente interrelacionados. Orientar las emociones, y la motivación constante, para lograr los objetivos es fundamental para prestar atención, automotivarse, manejarse y realizar actividades creativas. Con el autocontrol emocional se puede llegar a dominar la impulsividad, lo cual, generalmente está presente en el logro de muchos objetivos. Los individuos que poseen estas habilidades suelen ser más productivas y efectivas en las actividades que se proponen.

4. Reconocer las emociones de los demás: Una virtud fundamental es la empatía, que no es otra cosa que el conocimiento de las propias emociones. La empatía es el cimiento del altruismo. Las personas empáticas sincronizan mejor con las señales sublimes que muestran lo que los demás necesitan o desean. Es una competencia emocional apropiada para las labores de ayuda y servicios en sentido amplio (profesores, orientadores, pedagogos, psicólogos, psicopedagogos, médicos, abogados, expertos en ventas, etc.).

5. Establecer relaciones: La habilidad de mantener buenas relaciones con los demás es, en gran medida, saber manejar las emociones de las personas. La competencia social y las habilidades que posee, son la base del liderazgo, popularidad y eficiencia interpersonal. Aquellos que logran dominar estas habilidades sociales tienen la capacidad de interactuar de forma sutil y efectiva con los demás.

\section{La Inteligencia Emocional en los Actores Educativos}

Dentro de las instituciones educativas es importante hacer estudios que tengan que ver con la capacidad de dirigir, orientar y fortalecer las relaciones de los actores educativos tanto en lo personal como en lo social para que, según Gonzáles (2008), "se pueda mejorar los esfuerzos colectivos, hacia una continua innovación y adaptación a las nuevas exigencias organizacionales" (p.34). Ciertamente, con la inteligencia emocional se pueden generar en los seres 


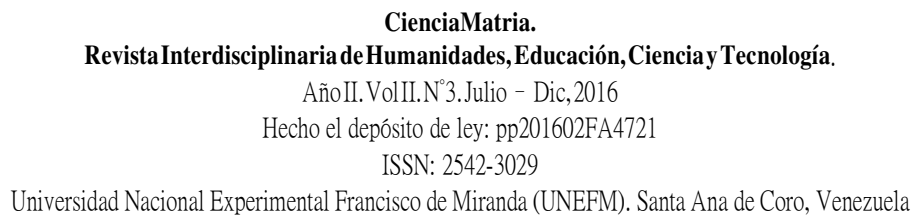

humanos cambios relevante en cuanto al conocimiento de sus propias emociones y la capacidad de guiar y avanzar en el desarrollo de las capacidades de cualquier individuo con mayor eficiencia.

Por su parte, Goleman (2004) define, inteligencia emocional, como "la capacidad de reconocer los sentimientos propios y ajenos, y el poder automoverse para manejar positivamente las emociones internas y las relaciones con los demás" (p.56). Es decir, la inteligencia emocional permite que una persona esté consciente de los sentimientos que esté experimentando en ese momento, dándole la oportunidad al individuo de reconocer, manejar y controlar sus propios estados internos. En esa auto-reflexión, denominada así por Goleman (ob cit), "la mente observa, investiga las experiencias mismas, incluidas tanto las emociones como la conciencia de sí mismo, para conservar la auto-reflexión" (p.58).

Así que, para alcanzar la inteligencia emocional, no sólo se necesita conocer lo que se quiere para lograrlo, sino que es importante ponerlo en práctica, para obtener con ello, ciertas competencias emocionales. En ese sentido, Gonzáles (2008) plantea que "las personas que muestran una adecuada competencia emocional, disfrutan de una situación ventajosa en los diversos dominios y dimensiones de la vida, por ello se sienten más satisfechos consigo mismos" (p.34), es decir, los individuos tienden a ser más eficaces en cualquier trabajo en el que se desenvuelvan.

Por otro lado, Salovey y Mayer (1999) señalan que la inteligencia emocional es "la capacidad que tiene un individuo de monitorear, así como el de regular los sentimientos propios y ajenos, utilizando sus emociones para guiar el pensamiento de las acción" (p.12).

Cortese (2003) agrega que el término de inteligencia emocional se refiere a "la capacidad humana de sentir, entender, controlar y modificar estados emocionales propios y en los demás, es decir, implica dirigir y así como equilibrar las emociones" (p.25). En este orden de ideas, hay que destacar que la inteligencia emocional es un talento que cualquier persona o actor educativo, debe determinar 
sí, en primer lugar, lo posee; y en segundo lugar, sí la posee, debe saber controlarla, para así liderar de forma eficaz cualquier organización o institución educativa.

González (2008) expresa que la inteligencia emocional es:

La capacidad que tiene el individuo para monitorear las emociones propias y de un colectivo, con incidencia en el pensamiento y la acción e implica un estado psicológico de sentir, así como de modificar, emociones para lograr el equilibrio (p.35).

Es importante destacar que todos los actores educativos deban poseer un liderazgo basado en las habilidades emocionales, que sean dignos de confianza para lograr acuerdos con los demás, en otras palabras, debe saber escuchar, persuadir y aconsejar, para llegar a tener un clima eficaz dentro de la institución educativa. Por otro lado, Grajales (2006), explica de forma pragmática que:

Los individuos con altos niveles de inteligencia emocional son más exitosos, por ende, viven una vida más plena y feliz. En el trabajo disfrutan del respeto de sus colegas, subordinados; resultando ser promovidos con más rapidez; son poseedores de un sistema inmune más fuerte, por lo tanto disfrutan de una buena salud (p.35).

Para que esto sea así, es importante mencionar que todo va a depender del tipo de relación que tenga la persona consigo misma y la forma en que se relacione con los demás, además, también es importante, el tipo de liderazgo que mantenga en la institución y la habilidad para trabajar en equipo. Al poseer efectivamente estos elementos dentro de las organizaciones educativas, se garantiza el logro del éxito en cuanto a las relaciones sociales, que sin duda alguna, es una de las más importantes en el mundo laboral. En ese sentido, cunado los actores educativos, poseen diversas cualidades emocionales, su participación en el ámbito laboral, es activa y con disposición de trabajar en equipo, sin embargo, debe mantenerse en contacto con las corrientes emocionales que se desprenden del colectivo para que tenga la oportunidad de detectar a tiempo los efectos de sus decisiones y los sentimientos no expresados por ellos. 
Para Cooper y Sawaf (2004), la inteligencia emocional es "la capacidad de sentir, entender, y aplicar el poder de la agudeza de las emociones como fuente de energía humana, conexión e influencia" (p.3), es decir, las emociones son fuentes de empuje que guían el comportamiento de los actores educativos hacia la socialización, para así mejorar de forma continua el clima dentro de las instituciones educativas, por lo que se espera que posean aptitudes cognitivas y sobre todo aptitudes emocionales.

Gonzáles (2008) también define la inteligencia emocional como "la capacidad que deben poseer las personas para dominar equilibradamente sus emociones, tanto de orden interno como externo, observando a través de un comportamiento adecuado, en atención al contexto y en su gente" (p.37). Para lograr esto, se requiere que los actores educativos sean personas altamente inteligentes, no sólo por poseer inteligencia intelectual, sino además por poseer, desarrollar y aplicar su inteligencia emocional.

\section{Competencias Emocionales}

Existe una gran variedad de definiciones sobre competencias emocionales, los diferentes autores no han podido llegar a un acuerdo único sobre cuál debería ser su designación, para algunos expertos es competencia emocional, otros prefieren utilizar la designación de competencia socio-emocional; otro autores prefieren utilizar el plural y denominarlo competencias emocionales o competencias socioemocionales. Lo cierto es que, se han elaborado algunas propuestas que tratan de describirlo, como por ejemplo:

Goleman (1995) describe cinco dominios de competencias emocionales a saber: "autoconciencia emocional; manejo de las emociones, automotivación, empatía y habilidades sociales" (p.29). Asímismo Salovey y Sluter (1997) identificaron cinco dimensiones básicas en las competencias emocionales: "Cooperación; asertividad, responsabilidad, empatía y autocontrol" (p.11). 


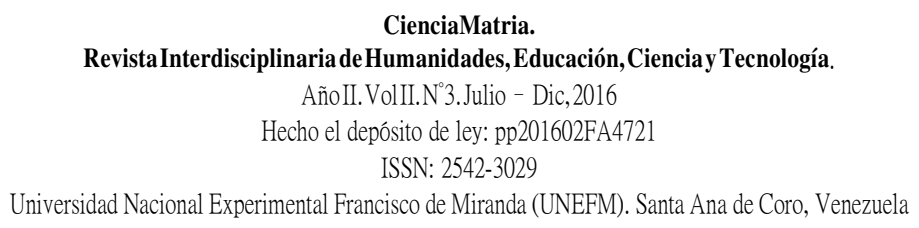

Siete años más tarde, Goleman, Boyatsis y Mckee (2002) proponen cuatro dominios con diecinueve competencias (p.72-73)

1. Conciencia de uno mismo: conciencia emocional de uno mismo, valoración adecuada de uno mismo y confianza en uno mismo.

2. Autogestión: autogestión emocional, Transparencia, adaptabilidad, logro, iniciativa y optimismo.

3. Conciencia social: empatía, conciencia de la organización y servicio.

4. Gestión de las relaciones: Liderazgo inspirado, influencia, desarrollo de los demás, catalizar el cambio, gestión de los conflictos, establecer vínculos y trabajo en equipo y colaboración.

Para Saarni (2000) la competencia emocional se vincula con la autoeficacia al expresar emociones en los intercambios sociales. Por lo tanto la autoeficacia es "la capacidad y las habilidades que tiene el individuo para lograr los objetivos deseados" (p.68), es decir, para que haya autoeficacia es necesario que el individuo tenga conocimiento de sus propias emociones y la capacidad de controlarlas para lograr los resultados esperados y esto dependerá de los principios morales y los valores éticos que posea una persona, los cuales, sin duda alguna, influyen en las respuestas emocionales, promoviendo así la integridad del individuo.

Además, Saarni (2000) presenta el siguiente listado de habilidades de la competencia emocional:

Conciencia del propio estado emocional; habilidad para discernir las habilidades de los demás; habilidad para utilizar el vocabulario emocional y términos expresivos habitualmente en una cultura; capacidad para implicarse empáticamente; habilidad para comprender que el estado emocional interno no necesita corresponder con la expresión externa; tanto en uno mismo como en los demás; habilidad para afrontar emociones negativas mediante la utilización de estrategias de auto control; conciencia de la estructura y naturaleza de las relaciones vienen en parte definida por: el grado de inmediatez emocional o sinceridad expresiva y el grado de reciprocidad o simetría en la relación; capacidad de autoeficacia emocional (p.77-78). 
Las competencias socio-emocionales propuestas por Graczyk (2000), Payton (2000) y CASEL (2006) se pueden resumir de la siguiente manera: Toma de conciencia en los sentimientos, manejo de los sentimientos, tener en cuenta la perspectiva, análisis de normas sociales, sentido constructivo del yo, Responsabilidad, cuidado, Respeto por los demás, identificación del problema, fijar objetivos adaptativos, solución del problema, comunicación receptiva, comunicación expresiva, cooperación, negociación, negativa y buscar ayuda.

Bisquerra (2003) señala que las competencias emocionales se dividen en cuatro bloques, y para efectos de esta investigación, se tomará en cuenta la estructuración completa de las competencias emocionales propuestas por este autor.

1. Conciencia emocional: Capacidad para tomar conciencia de las propias emociones y de las emociones de los demás, incluyendo la habilidad para captar el clima emocional de un contexto determinado. (p.23)

(a) Toma de conciencia de las propias emociones: capacidad para percibir con precisión los propios sentimientos y emociones; identificarlos y etiquetarlos.

(b) Dar nombre a las emociones: Eficacia en el uso del vocabulario emocional adecuado y las expresiones disponibles en un contexto cultural determinado.

(c) Comprensión de las emociones: de los demás e implicarse empáticamente en sus vivencias emocionales. Incluye la pericia de servirse de las claves situacionales y expresivas (comunicación verbal y no verbal) que tienen un cierto grado de consenso cultural para el significado emocional.

2. Regulación emocional: Capacidad para manejar las emociones de forma apropiada. Supone tomar conciencia de la relación entre emoción, cognición y comportamiento y tener buenas estrategias de afrontamiento o capacidad para auto generarse emociones positivas (p.23-24).

(a) Tomar conciencia de la interacción entre emoción, cognición y comportamiento: Los estados emocionales inciden en el comportamiento y éstos 


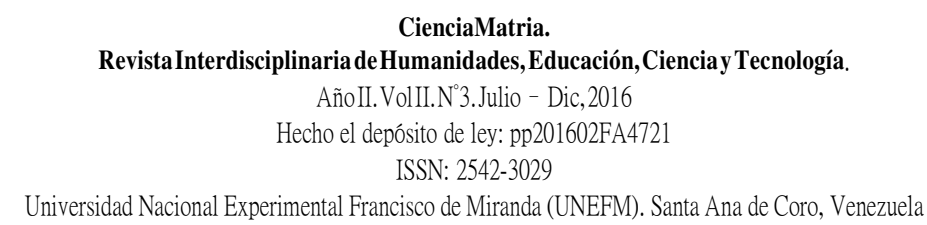

en la emoción; ambos pueden regularse por la cognición (razonamiento, conciencia).

(b) Expresión emocional: capacidad para expresar las emociones de forma apropiada y comprender que el estado emocional interno no necesita corresponder con la expresión externa que de él se presenta, tanto en uno mismo como en los demás. En niveles de mayor madurez, comprensión del impacto de la propia expresión emocional en otros, y facilidad para tenerlo en cuenta en la forma de mostrarse a sí mismo y a los demás.

(c) Regulación emocional: Incluye la regulación de la impulsividad (ira, violencia, comportamientos de riesgo), tolerancia a la frustración para prevenir estados emocionales negativos (ira, estrés, ansiedad, depresión) y perseverar en el logro de los objetivos a pesar de las dificultades; capacidad para diferir recompensas inmediatas a favor de otras más a largo plazo pero de orden superior.

(d) Habilidad para afrontar emociones negativas: mediante la utilización de estrategias de autorregulación que mejoren la intensidad y la duración de tales estados emocionales.

(e) Competencia para auto generar emociones positivas: (alegría, amor, humor, fluir) y disfrutar de la vida. Capacidad para auto-gestionar el propio bienestar subjetivo en busca de una mejor calidad de vida.

3. Autonomía emocional: Concepto amplio que incluye un conjunto de características y elementos relacionados con la autogestión personal, entre las que se encuentran la autoestima, actitud positiva ante la vida, responsabilidad, capacidad para analizar críticamente las normas sociales, la capacidad para buscar ayuda y recursos, así como la auto eficacia emocional (p.24-25).

(a) Autoestima: tener una imagen positiva de sí mismo; estar satisfecho de sí mismo; mantener buenas relaciones consigo mismo.

(b) Auto motivación: capacidad de auto motivarse e implicarse emocionalmente en actividades diversas de la vida personal, social, profesional o de tiempo libre. 
(c) Actitud positiva: capacidad para tener una actitud positiva ante la vida. Sentido constructivo del yo (self) y de la sociedad; sentirse optimista y potente (empowered) al afrontar los retos diarios.

(d) Responsabilidad: intención de implicarse en comportamientos seguros, saludables y éticos y asumir las consecuencias de las decisiones tomadas.

(f) Auto-eficacia emocional: capacidad de auto-eficacia emocional. Significa que uno acepta su propia experiencia emocional, tanto si es única y excéntrica como si es culturalmente convencional, y esta aceptación está de acuerdo con las creencias del individuo sobre lo que constituye un balance emocional deseable. En esencia, uno vive de acuerdo con su "teoría personal sobre las emociones" cuando demuestra auto-eficacia emocional que está en consonancia con los propios valores morales.

(g) Análisis crítico de normas sociales: capacidad para evaluar críticamente los mensajes sociales, culturales relativos a normas sociales y comportamientos personales.

(h) Resistencia: para afrontar las situaciones adversas que la vida pueda deparar.

4. Competencia social: Capacidad para mantener buenas relaciones con otras personas. Esto implica dominar las habilidades sociales, capacidad para la comunicación efectiva, respeto, actitudes pro-sociales, asertividad. (p.25)

(a) Dominar las habilidades sociales básicas: escuchar, saludar, despedirse, dar las gracias, pedir un favor, manifestar agradecimiento, pedir disculpas, mantener una actitud dialogante.

(b) Respeto por los demás: intención de aceptar y apreciar las diferencias individuales y grupales y valorar los derechos de todas las personas.

Practicar la comunicación receptiva: capacidad para atender a los demás tanto en la comunicación verbal como no verbal para recibir los mensajes con precisión.

(c) Practicar la comunicación expresiva: capacidad para iniciar y mantener conversaciones, expresar los propios pensamientos y sentimientos con claridad, 
tanto en comunicación verbal como no verbal, y demostrar a los demás que han sido bien comprendidos.

(d) Compartir emociones: conciencia de que la estructura y naturaleza de las relaciones vienen en parte definidas tanto por el grado de inmediatez emocional o sinceridad expresiva como por el grado de reciprocidad o simetría en la relación.

(e) Comportamiento pro-social y cooperación: capacidad para aguardar turno; compartir en situaciones diádicas y de grupo; mantener actitudes de amabilidad y respeto a los demás.

(f) Asertividad: mantener un comportamiento equilibrado, entre la agresividad y la pasividad. Esto implica la capacidad para defender y expresar los propios derechos, opiniones y sentimientos; decir "no" claramente y mantenerlo; hacer frente a la presión de grupo y evitar situaciones en las cuales uno puede verse coaccionado; demorar actuar o tomar decisiones en estas circunstancias de presión hasta sentirse adecuadamente preparado.

(g) Prevención y solución de conflictos: capacidad para identificar, anticiparse o afrontar resolutivamente conflictos sociales y problemas interpersonales. Implica la capacidad para identificar situaciones que requieren una solución o decisión preventiva y evaluar riesgos, barreras y recursos. Cuando inevitablemente se producen los conflictos, afrontarlos de forma positiva, aportando soluciones informadas y constructivas. La capacidad de negociación es un aspecto importante, que contempla una resolución pacífica, considerando la perspectiva y los sentimientos de los demás.

(h) Capacidad de gestionar situaciones emocionales o capacidad para inducir o regular las emociones en los demás.

5. Competencias para la vida y bienestar: Las competencias para la vida y el bienestar son la capacidad para adoptar comportamientos apropiados y responsables para afrontar satisfactoriamente los desafíos diarios de la vida, ya sean personales, profesionales, familiares, sociales, de tiempo libre, etc. Las 
competencias para la vida permiten organizar nuestra vida de forma sana y equilibrada, facilitándonos experiencias de satisfacción o bienestar. (p.25-26)

(a) Fijar objetivos adaptativos: Es la capacidad para fijar objetivos positivos y realistas. Algunos a corto plazo (para un día, semana, mes); otros a largo plazo (un año, varios años).

(b) Toma de decisiones: Desarrollar mecanismos personales para tomar decisiones sin dilación en situaciones personales, familiares, académicas, profesionales, sociales y de tiempo libre, que acontecen en la vida diaria. Supone asumir la responsabilidad por las propias decisiones, tomando en consideración aspectos éticos, sociales y de seguridad.

(c) Buscar ayuda y recursos: Es la capacidad para identificar la necesidad de apoyo y asistencia y saber acceder a los recursos disponibles apropiados. Ciudadanía activa, participativa, crítica, responsable y comprometida.- Lo cual implica reconocimiento de los propios derechos y deberes; desarrollo de un sentimiento de pertenencia; participación efectiva en un sistema democrático; solidaridad y compromiso; ejercicio de valores cívicos; respeto por los valores multiculturales y la diversidad, etc. Esta ciudadanía se desarrolla a partir del contexto local, pero se abre a contextos más amplios (autonómico, estatal, europeo, internacional, global). Las competencias emocionales son esenciales en la educación para la ciudadanía (Bisquerra, 2008).

(d) Bienestar emocional: Es la capacidad para gozar de forma consciente de bienestar (emocional, subjetivo, personal, psicológico) y procurar transmitirlo a las personas con las que se interactúa. Adoptar una actitud favorable al bienestar. Aceptar el derecho y el deber de buscar el propio bienestar, ya que con ello se puede contribuir activamente al bienestar de la comunidad en la que uno vive (familia, amigos, sociedad).

(e) Fluir: Capacidad para generar experiencias óptimas en la vida profesional, personal y social. 
Por tanto, Bisquerra (2003) define las competencias emocionales como el "conjunto de conocimientos, capacidades, habilidades y actitudes necesarias para comprender, expresar y regular la forma apropiada los fenómenos emocionales" (p.21), es decir, las competencias emocionales fomentan los procesos de aprendizajes, las relaciones interpersonales, la solución de los problemas la consecución y mantenimiento de un trabajo.

En las personas racionales, las emociones no solo tiene un papel más determinante en la toma de decisiones, sino que además el individuo puede tener el control de la impulsividad, persistencia, motivación, empatía y destrezas sociales. Goleman (1995) defiende el planteamiento en el que se afirma que existen dos mentes, una racional y una emocional, y que las formas de conocimientos son distintas para cada caso y cuando trabajan de forma concatenada, interconectando los diferentes conocimientos, la inteligencia emocional crece al igual que la capacidad intelectual.

\section{Teoría Emergente Intersubjetiva de la Emocionalidad para la Formación Universitaria por Competencias}

En el apartado anterior se definió una categoría y subcategoría o conceptos que han sido fundamentadas desde la óptica de los informantes claves, sobre la inteligencia emocional y la innovación curricular en el Programa de Ingeniería Agronómica de la UNEFM. Ahora, en esta parte, se proyecta una teoría emergente intersubjetiva donde se gestione el papel que esta categoría juega en la configuración de la formación universitaria por competencias.

A continuación se presenta un diagrama para explicar la teoría emergente intersubjetiva para la formación universitaria por competencias: 


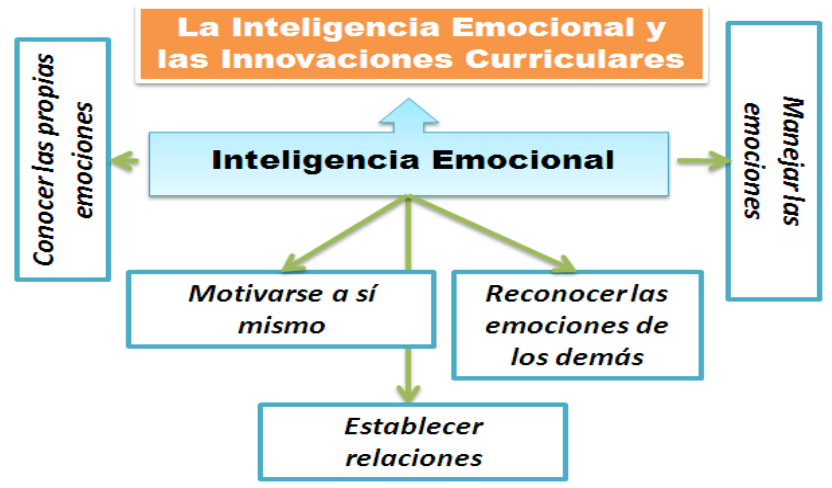

Gráfico 1: Teoría Emergente Intersubjetiva para la Formación Universitaria por Competencias. Adaptado por la Investigadora

La formación universitaria por competencias como innovación curricular, es un enfoque que propone una educación flexible que vincula el ámbito educativo con el sector productivo y debe ser entendida como un enfoque donde, se le dé mayor importancia a la crítica y profundidad de los contenidos, cuya intención no es solo orientar los conocimientos a la producción, sino a aquellos que articulan una concepción del ser, del saber, del hacer y del saber convivir que los ayudará a desenvolverse en una determinada situación dentro de la sociedad o específicamente, en el campo de trabajo.

Todo esto, propicia el cambio educativo que se genera mediante la reflexión y la formación de directivos y docentes, de esta reflexión y formación es donde la inteligencia emocional entra en juego, haciendo que los afectados en el cambio curricular acepten o rechacen la propuesta de innovación o transformación, por lo tanto, es importante saber manejar las emociones de quienes recae la responsabilidad de llevar a cabo, implementar y aplicar un cambio curricular.

La emoción forma parte de cada persona y desempeña un papel muy importante en la manera de actuar, en el aprendizaje, creatividad e interacción social, las emociones y la inteligencia emocional están asociados a las experiencias que vive una persona, la acción que ejecuta un individuo ante una determinada situación es lo que se conoce como emoción y la manera cómo 
reacciona, reconociendo esa emoción, es lo que se conoce como Inteligencia Emocional.

En una formación universitaria, la inteligencia emocional y el manejo de la misma es indispensable, por lo tanto, es importante que el que dirige, oriente y fortalece las relaciones de los actores educativos, tanto en lo personal como en lo social, debe ser capaz de manejar los esfuerzos colectivos hacia una continua innovación y adaptación a las exigencias sociales. Además, los docentes y estudiantes con inteligencia emocional, aprenden y se adaptan rápida y constantemente a cualquier situación o cambio que se genere en su cotidianidad. Aprender a conocer, manejar y controlar sus emociones, es un proceso funcional que permite adaptarse a los cambios curriculares. Cuando se aprende a reconocer las emociones de sí mismos y en los demás se hace más fácil controlarlas para producir las motivaciones que se necesita para enfrentar y dar respuesta con responsabilidad a las diversas situaciones de la vida, que de otra forma serían complicadas de entender.

\section{CONSIDERACIONES FINALES}

La inteligencia emocional y el manejo de la misma es indispensable, por lo tanto, es importante que el que dirige, oriente y fortalece las relaciones de los actores educativos, tanto en lo personal como en lo social, debe ser capaz de manejar los esfuerzos colectivos hacia una continua innovación y adaptación a las exigencias sociales. Además, los docentes y estudiantes con inteligencia emocional, aprenden y se adaptan rápida y constantemente a cualquier situación o cambio que se genere en su cotidianidad. Aprender a conocer, manejar y controlar sus emociones, es un proceso funcional que permite adaptarse a los cambios curriculares. Cuando se aprende a reconocer las emociones de sí mismos y en los demás se hace más fácil controlarlas para producir las motivaciones que se necesita para enfrentar $y$ dar respuesta con responsabilidad a las diversas situaciones de la vida, que de otra forma serían complicadas de entender. 
Todos los actores educativos deben poseer un liderazgo ligado a las habilidades emocionales, además, que sean dignos de confianza para lograr acuerdos con los demás, es decir, deben saber escuchar, persuadir y aconsejar, para llegar a tener un clima eficaz dentro de la institución educativa.

Cuando un individuo tiene la capacidad de reconocer sus propias emociones, puede lograr manejar y controlar diversas situaciones sin que éstas afecten, de forma errónea, su estado emocional.

El manejo de las emociones tiene que ver con la regulación de la impulsividad, tolerancia a la frustración para prevenir emocionalidades negativas y perseverar así el logro de las metas a pesar de las dificultades.

Con autocontrol emocional se puede llegar a dominar el ser impulsivo, lo cual, generalmente se encuentra presente cuando se quiere lograr cumplir con los objetivos. Los individuos que poseen las habilidades de autocontrol, automotivación, suelen ser eficaces y conseguir lo que se proponen en las actividades que se asignan.

Reconocer las emociones es una herramienta que los individuos pueden utilizar para establecer lazos que permitan un acercamiento con los demás y con ello lograr controlar las situaciones que afecten la estabilidad emocional del colectivo.

El establecimiento de relaciones viene dado por las habilidades sociales básicas que posea una persona, la manera como acepta y aprecia las diferencias individuales y grupales y la forma como valora los derechos de todas las personas.

\section{REFERENCIAS BIBLIOGRÁFICAS}

Bisquerra, R. (2003). Educación emocional y competencias básicas para la vida. Revista de Investigación Educativa, 21(1), 7-43.

Bisquerra (2008) Educación para la Ciudadanía y Convivencia. El Enfoque de la Educación Emocional. Wolters Kluwer. Barcelona.

Humanas.Maracaibo Venezuela. 


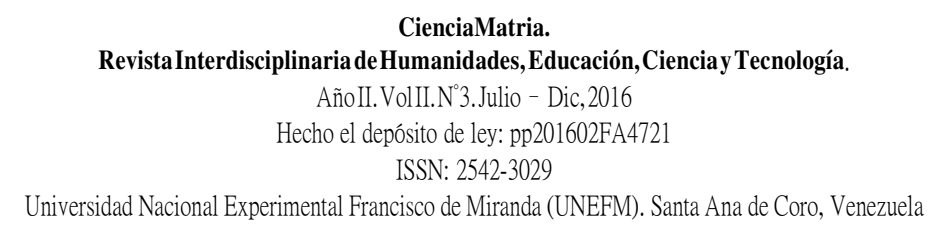

CASEL (Collaborative for Academic, Social, and Emotional Learning). (2006) SEL Competences [en línia] (UIC) University of Illinois at Chicago: Chicago.

Cooper y Sawaf (2004) Inteligencia Emocional Aplicado al Liderazgo y a las Organizaciones. Ediciones Norma. Colombia.

Cortese, (2003). La Inteligencia Emocional en la empresa. Disponible en www.sht.com.ar. Extraido el 28-04.07 por Perozo (2012)

Goleman (1995) Inteligencia Emocional. Karios. Barcelona

Goleman (2002) El Liderazgo Resonante. México. Edit Mc Graw Hill

Goleman (2004) El Liderazgo Resonante Crea Más. Caracas. Edit Melvin C.A

Goleman, Boyatzis, Mckee, (2004). El Líder Resonante Crea Más. El Poder de la Inteligencia Emocional, Editorial Melvin C. A. Venezuela.

Goleman, D. (2009): Introducción. En L. Lantier, Inteligencia Emocional Infantil y Juvenil. Madrid. Editorial Aguilar.

Gonzáles (2008) La Inteligencia Emocional en el Liderazgo Gerencial del Director de Educación Media Diversificada y Profesional. Luz. Venezuela.

Grajales (2006) Inteligencia Emocional. México. Edit paidos.

Payton, Graczyk, (2000). Social and emotional learning: A framework for promoting mental health and reducing risk behaviors in children and youth (download). Journal of School Health, 70, 179-185.

Pereda (2008). Perfil del docente en el siglo XXI, en el marco de un modelo educativo basado en competencias. MOPDF.com

Saarni, C. (2000). Emotional Competence. A Developmental Perspective. En R. Bar-On y J. D. A. Parker (Eds.), The Handbook of Emotional

Intelligence.Theory, Development, Assessment, and Application at Home, School, and in the Workplac. San Francisco, Ca: Jossey-Bass, pp 68-91.

Salovey y Mayer (1990) Inteligencia Emocional. Imaginación, Cognición y Personalidad. 
Salovey y Mayer (1997). What is Emotional Intelligence? En P. Salovey \& D.J.Sluyter (Eds.), Emotional development and emotional intelligence: Educational implications (pp. 3-31). Nueva York: Basic Books.

Salovey, P., y Sluyter, D. J. (1997). Emotional Development and Emotional Intelligence. Educational Implications. Nueva York: Basic Books.

Salovey y Mayer (1999) Inteligencia Emocional. Vergara Editor. 